\title{
Stress Fracture of the Radial Styloid Process in a Judo Player: A Case Report
}

\author{
Hiroshi Hashiguchi ${ }^{1}$, Satoshi Iwashita ${ }^{1}$, Atsushi Ohkubo ${ }^{1}$, \\ Takuya Sawaizumi ${ }^{2}$ and Shinro Takai ${ }^{2}$ \\ ${ }^{1}$ Department of Orthopaedic Surgery, Nippon Medical School Chiba Hokusoh Hospital \\ ${ }^{2}$ Department of Orthopaedic Surgery, Nippon Medical School
}

\begin{abstract}
Stress fractures of the upper limbs are uncommon, and are most often reported as individual cases or small series. In particularly, stress fractures around the wrist are even less common. A stress fracture of the radial styloid process in a judo player was surgically treated, and a favorable treatment outcome was obtained. A 16-year-old adolescent boy experienced pain in the right wrist, with no apparent trigger, while playing judo. Stress fracture of the radial styloid process was diagnosed with plain radiographs and was treated conservatively with cast immobilization. Although bone union was achieved, the fracture recurred after he resumed paying judo. Thus, surgical treatment was performed. The procedure was resection of the distal bone fragment. He resumed practicing 2 months postoperatively and returned to judo matches after 1 more month. As of 1 year after distal bone fragment resection, he was able to participate in judo without pain, limited range of motion, or instability of the wrist.
\end{abstract}

(J Nippon Med Sch 2015; 82: 109-112)

Key words: stress fracture, radial styloid process, sports injury

\section{Introduction}

In athletes, stress fractures are thought to be caused by repetitive stress applied to a structure, as in running and other athletic activities. Stress fractures usually occur in the lower extremities, and the most commonly affected sites are in the legs and feet, such as the tibia and metatarsal bones, in track and field athletes ${ }^{1-3}$. On the other hand, stress fractures of the upper limbs are less common, and most reported fractures are around the shoulder and elbow. In particular, few stress fractures of the wrist have been reported ${ }^{4-6}$. We report on a judo player who sustained a stress fracture of the radial styloid process.

\section{Case Presentation}

A 16-year-old adolescent boy presented with a chief complaint of right wrist pain. The pain had occurred without a discernible trigger while he was playing judo. Because the pain gradually worsened, he visited our department 2 months after onset.

Examination at the initial visit showed that the range of motion in the wrist was normal, but that pain oc- curred with dorsiflexion of the wrist. Tenderness was noted on the radial styloid process. Grip strength was $29.8 \mathrm{~kg}$ on the right and $36.4 \mathrm{~kg}$ on the left. An anteroposterior radiograph showed a bone fragment suggestive of an avulsion fracture of the radial styloid process, and also showed a partially osteosclerotic lesion with unclear margins (Fig. 1). On the basis of the history of the present illness and the plain radiographic findings, a stress fracture was diagnosed; the patient was treated with cast immobilization and asked to discontinue sporting activities. After 1 month of immobilization, plain radiograph revealed favorable callus formation. The cast was, therefore, removed, and a prosthesis was applied for fixation. Two months later, a plain radiograph revealed bone union (Fig. 2), and the pain and tenderness had also resolved. Thus, muscle training was started, and the patient resumed judo 1 month later. However, 1 month after the patient resumed judo, the right wrist was forced into radial flexion during a judo match, and pain recurred. Plain radiographs obtained at our clinic revealed stress fracture separation of the radial styloid process, and functional radiography of the wrist showed displace-

Correspondence to Hiroshi Hashiguchi, MD, Department of Orthopaedic Surgery, Nippon Medical School Chiba Hokusoh Hospital, 1715 Kamagari, Inzai, Chiba 270-1694, Japan

E-mail: hashiguchi@nms.ac.jp

Journal Website (http://www.nms.ac.jp/jnms/) 
ment of the fracture site (Fig. 3). Magnetic resonance imaging of the distal bone fragment showed low signal intensity on T1-weighted images and high signal intensity on T2-weighted images (Fig. 4). In consideration of the symptoms, disease course, clinical findings, imaging findings, and the patient's desire for an early return to sports, he was determined to receive surgical treatment, and resection of the distal bone fragment was thus se-

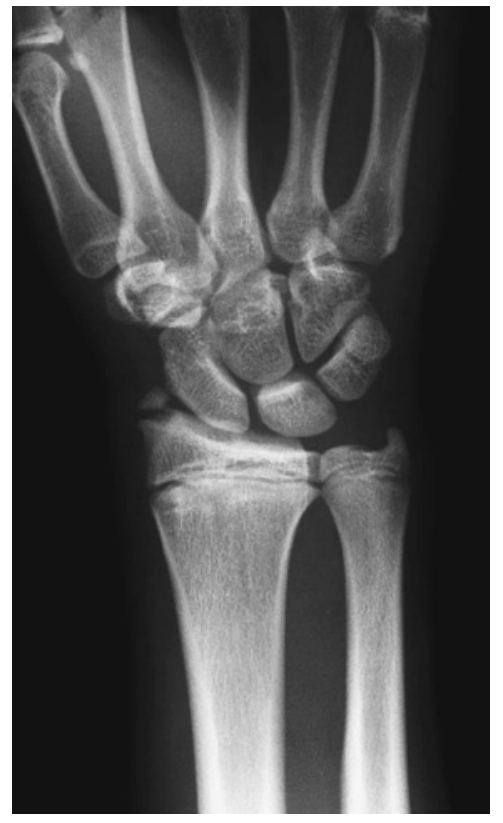

Fig. 1 Anteroposterior radiograph of the right wrist demonstrating a bone fragment of the radial styloid process.
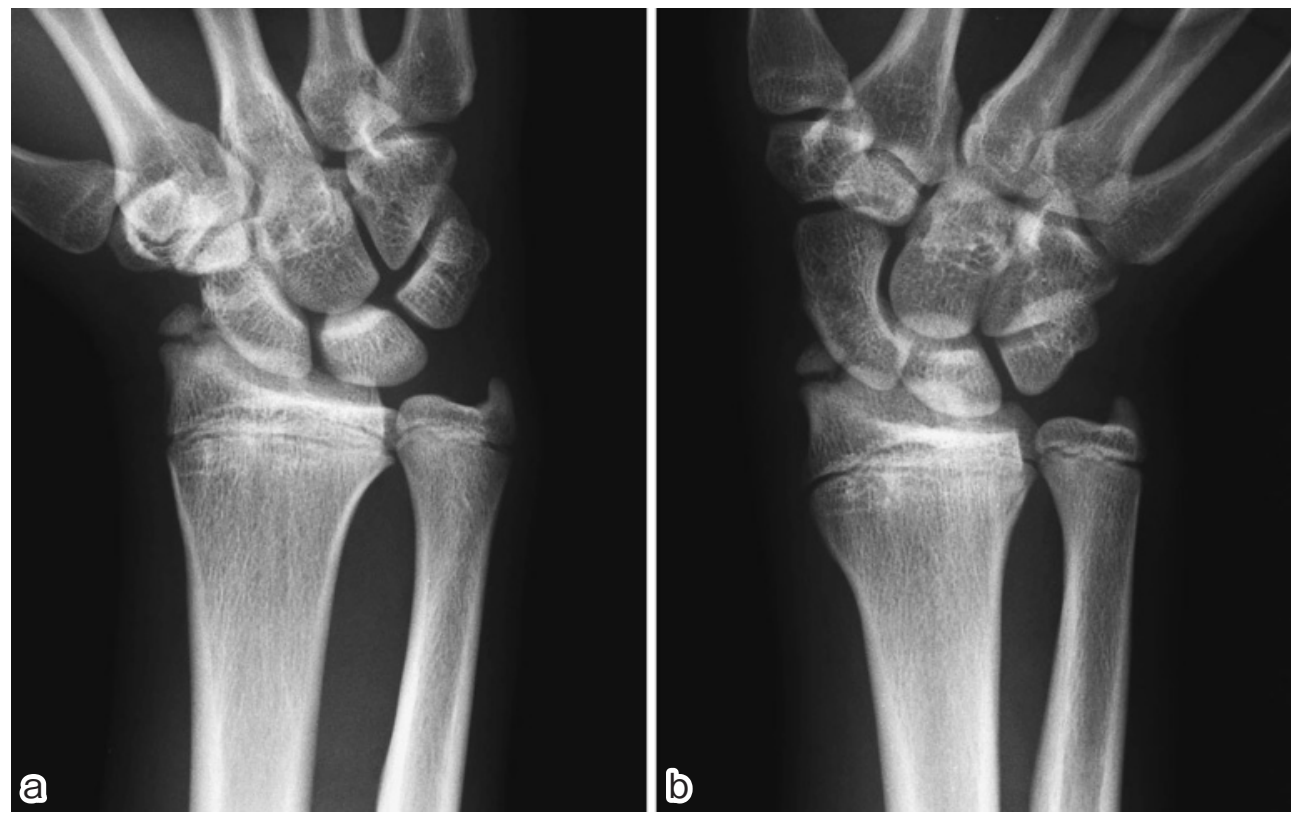

Fig. 3 Functional radiographs (a: radial deviation, b: ulnar deviation) demonstrating displacement of the fracture fragment. lected as the surgical procedure.

Arthroscopy indicated that the articular cartilage in the stress fracture site was completely separated (Fig. 5), and that the distal bone fragment was abnormally mobile. Surgery was performed with an approximately 2-cm skin incision made directly above the radial styloid process. Because some fibers of the external collateral ligament of the wrist were attached to the distal bone fragment, they

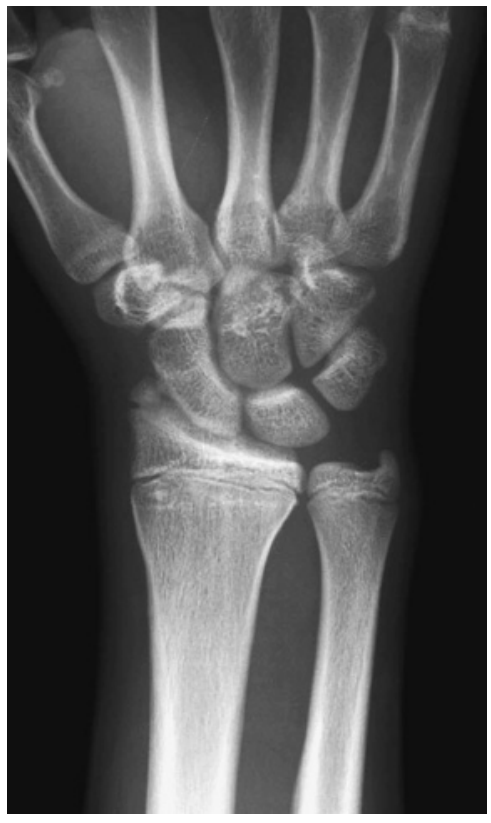

Fig. 2 Anteroposterior radiograph after 2 months of conservative treatment demonstrating bone union of the stress fracture. 


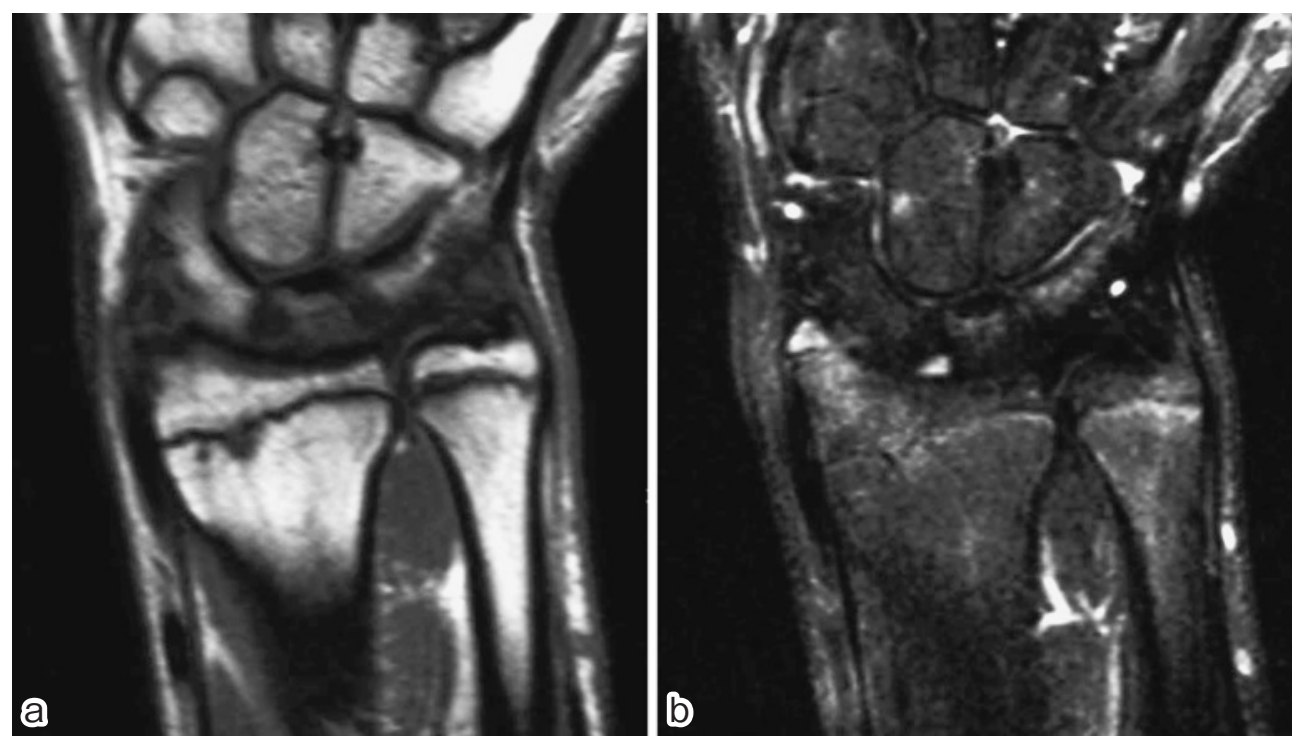

Fig. 4 Coronal magnetic resonance image demonstrating low signal intensity on T1-weighted images (a), and high signal intensity on T2-weighted images (b) in the distal bone fragment.

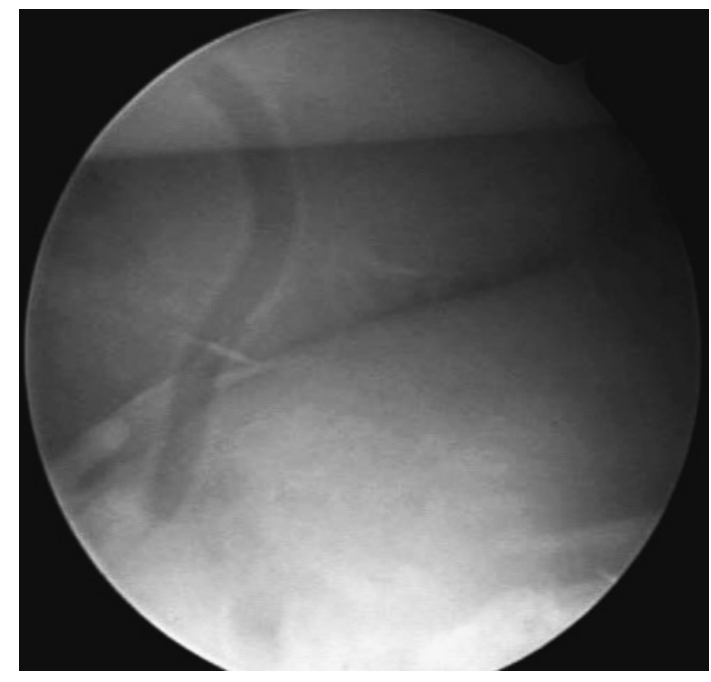

Fig. 5 Intraoperative arthroscopic image of the wrist demonstrating separation of the articular cartilage in the fracture site.

had to be resected to remove the distal bone fragment. The ligament fibers resected from the bone fragment were then immediately sutured to the remaining ligament. After the stability of the wrist was confirmed, the surgical wound was closed. Cast immobilization was applied after surgery. In postoperative week 3 , the cast was removed, and exercises for range of motion were started. In postoperative week 6 , muscle training was started. By postoperative week 8 , grip strength had recovered to $35.7 \mathrm{~kg}$ on the right and $37.9 \mathrm{~kg}$ on the left, and plain radiograph revealed no abnormalities (Fig. 6). Because no limitation in the range of motion of the wrist was ob-

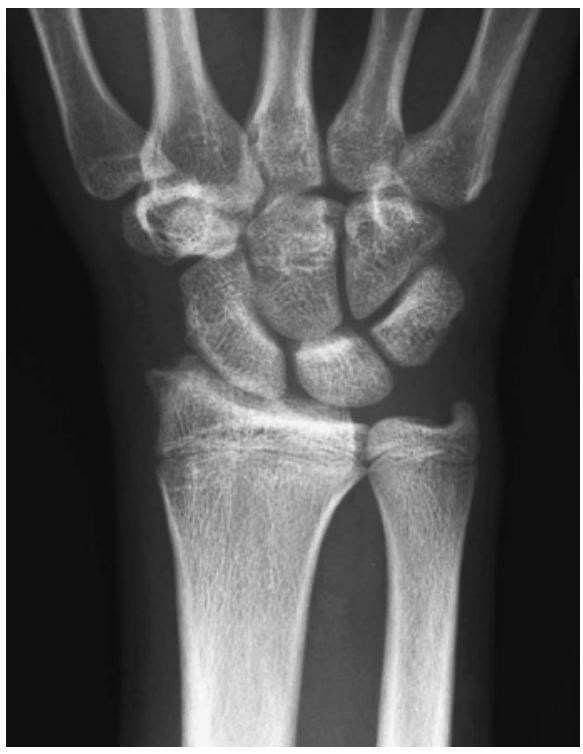

Fig. 6 Anteroposterior radiograph 8 weeks after surgery demonstrating no abnormalities.

served, the patient was allowed to resume judo practice. The patient returned to matches 3 months after surgery. As of 1 year after surgery, he was participating in judo without pain, range of motion limitation, or wrist instability.

\section{Discussion}

Initially, stress fractures were thought to be caused by the application of repetitive weight-bearing stress exceeding the allowable limit for bone. However, stress fractures reportedly occur even in the upper limbs, which are not 
subjected to weight-bearing stress ${ }^{7}$. Therefore, another widely accepted mechanism of stress fractures is bone microdamage caused by stress applied from various directions, such as compression, extension, flexion, and shearing due to muscle contraction ${ }^{8}$. Sinha et al. have classified patient with stress fractures of the upper limbs/ rib cage into 4 categories: 1) weight lifters (e.g., weight lifters and wrestlers), 2) upper extremity weight bearers (e.g., gymnasts and cheerleaders), 3) throwers (e.g., pitchers and javelin throwers), and 4) swingers (e.g., golfers and tennis players $)^{9}$. Because judo involves lifting the opponent with the upper limbs, as in wrestling, judo players may be classified as weight lifters, and stress fractures can occur anywhere in their upper limbs. The possible causes of stress fracture of the radial styloid process in judo include forced radial and ulnar flexion of the wrist in the pairing and throwing motions characteristic of judo. Forced radial flexion causes compression stress on the radial styloid process via the scaphoid, whereas forced ulnar flexion causes extension stress via the attached ligament. Our patient's favorite maneuver was a back throw, and he had practiced a back throw, which he had practiced occasionally with a heavyweight player, almost every day before onset of right wrist pain. The stress fracture might have been caused by repeated application of these stresses.

Although both conservative and surgical treatments were possible for our patient, conservative treatment and invasive osteosynthesis are both associated with greater risks of recurrence and failure of bone union, which would delay a patient's return to activity. Thus, given our patient's strong desire to resume playing judo, we believed that surgical resection of the distal bone fragment was the most appropriate treatment. Although resection of a bone fragment might induce instability of the wrist, we believe such instability is unlikely if repair of the ligament is performed as soon as possible and is followed by external fixation with a splint or prosthesis. Our patient returned to participating in judo matches 3 months after surgery, and year after surgery he had neither pain nor instability of the wrist.

In conclusion, for stress fractures of the radial styloid process sustained during sports, distal bone fragment resection and ligament repair are indicated for patients seeking an early return to sporting activities.

Conflict of Interest: The authors declare no conflict of interest.

\section{References}

1. Brukner $\mathrm{P}$, Bradshaw $\mathrm{C}$, Khan $\mathrm{K}$, White $\mathrm{S}$, Crossley $\mathrm{K}$ : Stress fractures: a review of 180 cases. Clin J Sports Med 1996; 6: 85-89.

2. Matheson GO, Clement DB, Mckenzie DC, Taunton JE, Lloyd-Smith DR, Macintyre JG: Stress fractures in athletes: a study of 320 cases. Am J Sports Med 1987; 15: 4657.

3. Wilson ES, Katz FN: Stress fractures: an analysis of 250 consecutive cases. Radiology 1969; 92: 481-486.

4. Fujioka H, Tsunemi K, Tanaka J: Bilateral stress fractures of the radial styloid process in a gymnast. J Hand Surg Eur 2010; 35: 243-244.

5. Bell RH, Hawkins RJ: Stress fracture of the distal ulna: a case report. Clin Orthop 1986; 209: 169-171.

6. Loosli AR, Leslie M: Stress fractures of the distal radius: a case report. Am J Sports Med 1991; 19: 523-524.

7. Brukner P: Stress fractures of the upper limbs. Sports Med 1998; 26: 415-424.

8. Schaffler MB, Radin EL, Burr DB: Mechanical and morphological effects of strain rate on fatigue of compact bone. Bone 1989; 10: 207-214.

9. Sinha AK, Kaeding CC, Wadley GM: Upper extremity stress fractures in athletes: clinical features of 44 cases. Clin J Sports Med 1999; 9: 199-202.

(Received, August 14, 2014)

(Accepted, September 22, 2014) 